\title{
miR-98 suppresses melanoma metastasis through a negative feedback loop with its target gene IL-6
}

\author{
Fei $\mathrm{Li}^{1}$, Xin-ji Li ${ }^{2}$, Li Qiao ${ }^{1}$, Fei Shi ${ }^{1}$, Wen $\mathrm{Liu}^{1}$, You Li ${ }^{1}$, Yu-ping Dang ${ }^{1}$, Wei-jie Gu${ }^{1}$, Xiao-gang Wang ${ }^{3,4}$ \\ and Wei Liu ${ }^{1}$
}

Dysregulated microRNA (miRNA) expression has a critical role in tumor development and metastasis. However, the mechanism by which miRNAs control melanoma metastasis is unknown. Here, we report reduced miR-98 expression in melanoma tissues with increasing tumor stage as well as metastasis; its expression is also negatively associated with melanoma patient survival. Furthermore, we demonstrate that miR-98 inhibits melanoma cell migration in vitro as well as metastatic tumor size in vivo. We also found that IL-6 is a target gene of miR-98, and IL-6 represses miR-98 levels via the Stat3-NF-KB-lin28B pathway. In an in vivo melanoma model, we demonstrate that miR-98 reduces melanoma metastasis and increases survival in part by reducing IL-6 levels; it also decreases Stat3 and p65 phosphorylation as well as lin28B mRNA levels. These results suggest that miR-98 inhibits melanoma metastasis in part through a novel miR-98-IL-6-negative feedback loop.

Experimental \& Molecular Medicine (2014) 46, e116; doi:10.1038/emm.2014.63; published online 3 October 2014

\section{INTRODUCTION}

The incidence of malignant melanoma of the skin (CMM) has continued to increase in the past 30 years despite aggressive education initiatives. ${ }^{1,2}$ Although most melanoma cases consist of localized disease that is curable with surgical resection, ${ }^{3,4}$ for those with regional or distant metastases, the prognosis is poor with a 10 -year survival rate of only $64 \%$ and $16 \%$, respectively. ${ }^{5}$ The poor prognosis and treatment for these patients is due in part to limited knowledge of melanoma progression and invasion.

Several studies have reported on the roles of microRNAs (miRNAs), which are small ( $\sim 22$ nucleotides), single-stranded, noncoding RNAs that induce posttranscriptional silencing of target genes through binding to $3^{\prime}$-untranslated regions (3'UTRs), in the progression of malignant melanoma. ${ }^{6-10}$ For example, Lu et al. ${ }^{8}$ observed differential miRNA expression that corresponded with tumor differentiation. In addition, Zhang et al. ${ }^{9}$ reported altered DNA copy number of miRNA genes in melanoma. Subsequent analysis by Mueller et al. ${ }^{10}$ revealed dysregulated miRNA expression in melanoma cell lines.

Roles for some miRNAs have also been noted in melanoma. Specifically, both miR- $137^{11}$ and miR-182 ${ }^{12}$ target the oncogene microphthalmia-associated transcription factor in mice. In addition, distant metastasis of melanoma cells was enhanced with miR-182 ${ }^{12}$ as well as with miR-214. ${ }^{13}$ Moreover, overexpression of miR-221/222 in melanoma cells increased their proliferation, invasion and chemotaxis in vivo, which was reversed in cells treated with antagonist $\operatorname{miR}-221 / 222 .^{10}$

Conversely, let-7b has been shown to be a negative regulator of melanoma cell growth via its effects on the cell cycle; it also inhibited anchorage-independent melanoma cell growth. ${ }^{14}$ Reduced melanoma metastasis in response to let-7b overexpression was also observed in an in vivo model of melanoma. ${ }^{15}$ However, the mechanism by which miRNAs influence cell migration in melanoma has yet to be determined.

The present study sought to test the hypothesis that miR-98 levels are associated with melanoma patient prognosis and influence melanoma cell migration and tumor growth by targeting IL-6 expression. Here, we found that miR-98 can inhibit melanoma cell migration in vitro and melanoma metastasis in vivo, suggesting that miR-98 may be a potential therapeutic target for melanoma metastasis.

${ }^{1}$ Department of Dermatology, The Air Force General Hospital of PLA, Beijing, China; ${ }^{2}$ Department of Radiation Oncology, The Air Force General Hospital of PLA, Beijing, China; ${ }^{3}$ Department of Cell Biology and Institute of Biomedicine, College of Life Science and Technology, Jinan University, Guangzhou, China and ${ }^{4}$ Jinan University and Hong Kong Baptist University Joint Laboratory of Innovative Drug Development, Jinan University, Guangzhou, China Correspondence: Professor X-g Wang, Department of Cell Biology and Institute of Biomedicine, Jinan University, No. 601 Huang-Pu Avenue West, Guangzhou 510632, China.

E-mail: txg_wang@jnu.edu.cn

or Dr W Liu, Department of Dermatology, The Air Force General Hospital of PLA, No. 30, Fu-Cheng Road, Beijing 100142, China.

E-mail: Iwei5811@126.com

Received 22 January 2014; revised 31 July 2014; accepted 8 August 2014 


\section{MATERIALS AND METHODS}

\section{Study participants}

Normal and tumor tissues were obtained from 48 patients and normal donors. Patient survival was followed for a median of 8 years. Informed consent was obtained from all patients. A melanoma tissue microarray with 208 tissue samples was purchased from US Biomax (Rockville, MD, USA). This study was approved by the institutional review board of The Air Force General Hospital of PLA (Beijing, China).

\section{Melanoma staging}

To analyze the relationship between miR-98 expression and melanoma progression, melanoma tissues of different stages were analyzed. Tissue stage was determined as described in Schuchter. ${ }^{16}$ After primary tumor $(\mathrm{T})$ staging thickness was measured, the tumors were classified into the following stages based on thickness: T1 $(<1 \mathrm{~mm})$, T2 (1.01$2 \mathrm{~mm}), \mathrm{T} 3(2.01-3 \mathrm{~mm})$ and $\mathrm{T} 4(>4 \mathrm{~mm})$.

\section{Quantitative reverse transcription-polymerase chain reaction analysis}

Total miRNA and mRNA were isolated from 20 normal (nevi) or 20 melanoma tissues or B16 cells using an miRNeasy FFPE Kit (Qiagen, Valencia, CA, USA) according to the manufacturer's instructions. To analyze the relationship between miR-98 expression and melanoma progression, melanoma tissues of different stages, including $13 \mathrm{~T} 1,11 \mathrm{~T} 2,9 \mathrm{~T} 3$ and $15 \mathrm{~T} 4$ tissues, were also analyzed. Furthermore, miR-98 levels were determined in 15 primary tumors and 15 metastatic tissues. Quantitative reverse transcriptionpolymerase chain reaction analyses for the miRNA and mRNA were performed using the One Step SYBRPrimeScript RT-PCR Kit II according to the manufacturer's instructions (Takara, Ohtsu, Japan). The following primers were used: let-7a sense, 5'-GCCGCTGAGGT AGTAGGTTGTGT-3', let-7b sense, 5'-GCCGCTGAGGTAGTAGG TTGTA-3', let-7c sense, 5'-GCCGCAGAGGTAGTAGGTTGC-3', let-7d sense, 5' -TGCCGGTGAGGTAGGAGG-3', let-7e sense, 5'-GCC CGTATCACAGCCAGCTT-3', let-7f sense, 5'-CGGCTGAGGTAGTA GATTGT-3' and let-7 antisense, 5'-GTGCAGGGTCCGAGGT-3' (all $120 \mathrm{bp}$ ); miR98 sense, 5'-CGGCTGAGGTAGTAGATTGT-3' and miR-98 antisense, 5'-GTCGTATCCAGTGCAGGGTCCGAGGTATTC GCACTGGATACGACAACAAT-3'; mmu-VEGFc sense, 5'-GAGGTC AAGGCTTTTGAAGGC- $3^{\prime}$ and antisense, $5^{\prime}$-CTGTCCTGGTATTGA GGGTGG-3' (160 bp); mmu-VEGFb sense, 5'-GCCAGACAGGGTTG CCATAC- $3^{\prime}$ and mmu-VEGFb antisense, 5'-GAGTGGGATGGATG ATGTCAG-3' (108 bp); mmu-VEGFa sense, 5'-CTGCCGTCCGATT GAGACC- $3^{\prime}$ and mmu-VEGFa antisense, $5^{\prime}$-CCCCTCCTTGTACCAC TGTC-3' (130 bp); mmu-E-cadherin sense, 5' -TCCAGGAACCTCCG TGATG-3' and mmu-E-cadherin antisense, 5'-GGGTAACTCTCTC GGTCCAG-3' (122 bp); mmu-N-cadherin sense, 5'-AGCCTGGGAC GTATGTGATG-3' and mmu-N-cadherin antisense, 5'-ATGTTGGGT GAAGGTGTGCT-3' (104 bp); mmu-Vimentin sense, 5'-CAAGTCCA AGTTTGCTGACCT-3' and mmu-Vimentin antisense, 5'-TCTTCC ATCTCACGCATCTG-3' (110 bp); mmu-Twist sense, 5'-CTCGGA CAAGCTGAGCAAG-3' and mmu-Twist antisense, 5'-ACGGAGAAG GCGTAGCTGAG-3' (126 bp); mmu-Snail sense, 5'-ACCCACACTGG TGAGAAGC- $3^{\prime}$ and mmu-Snail antisense, $5^{\prime}$-GACCAAGGCTGGAA GGAGTC-3' (92 bp); and lin28B sense, 5'-TCAGGATGGGAAGAG ATGCG-3' and lin28B antisense, 5'-TTCTCGCAGTTAACGCTGCT $-3^{\prime}$. Analysis of all let-7 subgroups were performed using the same let 7 reverse primer, which produced a product of $120 \mathrm{bp}$. The primers for mouse Stat3 (signal transducer and activator of transcription 3) were obtained from the qPCR Primer Assay Kit (SABiosciences/Qiagen, Valencia, CA, USA).

\section{Cell culture treatment and transfection}

B16-F1 mouse melanoma cells were purchased from ATCC (American Type Culture Collection, Manassas, VA, USA) and cultured in Dulbecco's modified Eagle's medium containing 10\% fetal bovine serum. For experiments using the nuclear factor $-\kappa \mathrm{B}(\mathrm{NF}-\kappa \mathrm{B})$ inhibitors BAY-117082 and JSH-23, cells were treated with IL-6 $\left(20 \mathrm{ng} \mathrm{ml}^{-1}\right)$ plus each inhibitor for $24 \mathrm{~h}$. Before transfection, cells were seeded in 6-well culture dishes. After $24 \mathrm{~h}$, the cells were transfected with $100 \mathrm{nM}$ miRNA-negative control (NC) or miR-98 (Ambion, Grand Island, NY, USA) using Lipofectamine RNAiMAX Transfection Reagent (Invitrogen, Carlsbad, CA, USA) for $36 \mathrm{~h}$, after which the transfection medium was replaced with normal growth medium. For transfection of siRNA-NC and siRNA-Stat3, Lipofectamine RNAiMAX Reagent (Invitrogen) was used according to the manufacturer's instructions. After $36 \mathrm{~h}$, the transfection medium was replaced with normal growth medium.

\section{Cell migration}

The CytoSelect 24-Well Cell Migration Assay Kit (Cell Biolabs, San Diego, CA, USA) was used to analyze B16-F1 cell migration after transfection of miRNA negative control (miRNA-NC) or miR-98 for $24 \mathrm{~h}$ or stimulation by IL- 6 for $36 \mathrm{~h}$ following the manufacturer's instructions. Cell migration was quantified and normalized to the mock transfection controls. The effects of miR-98 on cell migration after 0 and $12 \mathrm{~h}$ were also assessed using a CytoSelect 24-Well Wound Healing Assay Detection Kit (Cell Biolabs) according to the manufacturer's instructions.

\section{Animals}

All 80 male mice were handled in strict accordance with the Animal Welfare Assurance (permit no. kzdwsy2013117), and the experiments conformed to protocols approved by the Institutional Animal Care and Use Committee (IACUC) of the Air Force General Hospital of PLA. C57/B6J mice were obtained from the Vital River Laboratories Experimental Animal Technical (Beijing, China). There were a minimum of five mice per time point. Animals were kept in a 12-h light/dark cycle with food and water ad libitum. To establish a melanoma metastasis model to evaluate the effects of miR-98 or IL-6 on tumor metastasis, $5 \times 10^{6}$ B16-F1 cells transfected with miRNA-NC or miR-98 ( $n=8$ per group) were injected into the tail vein of the C57/B6J mice. Lung metastases were determined after 3 weeks. Tumor volume was also assessed at 12, 15, 18 and 21 days. For experiments using miR-98 mimics, animals were injected with $800 \mathrm{~nm}$ miR-98 mimics every 5 days. Metastatic tumor size and serum IL-6 levels were assessed on days 15, 20, 25, 30 and 35. Survival was also recorded. In addition, Stat 3 and p 65 phosphorylation and lin28B mRNA levels were measured on day 35 .

\section{IL-6 3'-UTR reporter assay}

B16-F1 cells were transfected with miRNA-NC, miR-98, anti-miRNANC or anti-miR-98 (Ambion), an miR-98 inhibitor, along with either an IL-6 luciferase reporter vector (Panomics, Santa Clara, CA, USA) or an miR-98-binding site mutant IL-6 reporter vector. Briefly, the $3^{\prime}$ UTRs of murine $I L-6$ was amplified by PCR from an MEF cell cDNA library and cloned downstream of the Renilla luciferase coding sequence into the pGL3 luciferase reporter vector (Promega, Madison, WI, USA). The IL-6 3-'UTR-Mut construct was generated by sitedirected mutagenesis using the QuickChange Mutagenesis Kit from 
Stratagene (La Jolla, CA, USA). Seed regions were mutated from UACCUCA to AUGGAGU. Reporter activity was determined after $24 \mathrm{~h}$ using the Dual-Luciferase Reporter Assay Kit (Promega) following the manufacturer's instructions.

\section{Western blot analysis}

Total protein extracts were obtained from B16-F1 cells. After centrifugation at $14000 \mathrm{~g}$, proteins were resolved by sodium dodecyl sulfate-polyacrylamide gel electrophoresis ( $20 \mu \mathrm{g}$ per lane) in a $10 \%$ polyacrylamide gel, transferred to polyvinylidene difluoride membranes and probed with primary antibodies specific for IL-6 (1:200; R\&D Systems, Minneapolis, MN, USA), lin28B (1:200; Santa Cruz Biotechnology, Santa Cruz, CA, USA), p-Stat3, Stat3, p-p65, p65 or glyceraldehyde 3-phosphate dehydrogenase (1:2000; Cell Signaling, Danvers, MA, USA). After incubation with the appropriate horseradish peroxidase-conjugated antibody, the proteins were visualized using the enhanced chemiluminescence western blot analysis system (Amersham Biosciences, Piscataway, NJ, USA).

\section{Enzyme-linked immunosorbent assay}

Cell culture supernatants were harvested from B16-F1 cells $36 \mathrm{~h}$ after transfection with miRNA-NC, miR-98, anti-miRNA-NC or anti-miR-98 as described above. IL-6 secretion was determined using an ELISA Kit (BD Biosciences, San Jose, CA, USA) following the manufacturer's instructions. For experiments using the IL-6 antibody (R\&D Systems), cells were treated with $50 \mathrm{ng} \mathrm{ml}$ IL-6 antibody for $12 \mathrm{~h}$.

\section{Immunohistochemistry}

IL-6 levels were assessed in a malignant melanoma tissue microarrays (Biomax) containing 128 primary malignant melanoma samples, 64 metastatic malignant melanoma samples, eight edges and normal tissues. Immunohistochemistry was performed in 5-mm sections of tissues that were formalin-fixed and paraffin-embedded. Reference sections were stained with hematoxylin and eosin (HE). The tissue sections were incubated with IL-6 primary antibodies (1:100 dilution; $\mathrm{R} \& \mathrm{D}$ Systems) at $4{ }^{\circ} \mathrm{C}$ overnight and then incubated with a horseradish peroxidase-conjugated mouse anti-rabbit secondary antibody (1:1000; R\&D Systems). Immunohistochemical analysis was performed using the 3,3'-Diaminobenzidine Kit (Sigma-Aldrich, St Louis, MO, USA).

\section{Chromatin immunoprecipitation}

Chromatin immunoprecipitation analysis was undertaken in B16-F1 cells that were treated with $20 \mathrm{ng}$ ml of IL-6 using a ChIP Kit (Abcam, Cambridge, MA, USA) following the manufacturer's instructions. After immunoprecipitation using a control immunoglobulin $G$ or an NF- $\mathrm{KB}$ antibody (Cell Signaling) and DNA purification, the immunoprecipitated DNA was amplified using the following primers that are specific for the NF- $\mathrm{-B}$ binding site in the $\operatorname{lin} 28 B$ promoter: sense, 5'-TCAGGATGGGAAGAGATGCG-3' and antisense, 5' ${ }^{\prime}$-TTCTCGCA GTTAACGCTGCT-3'.

\section{Statistical analysis}

Data were expressed as mean and standard deviation. Comparisons between two independent groups were performed using independent a
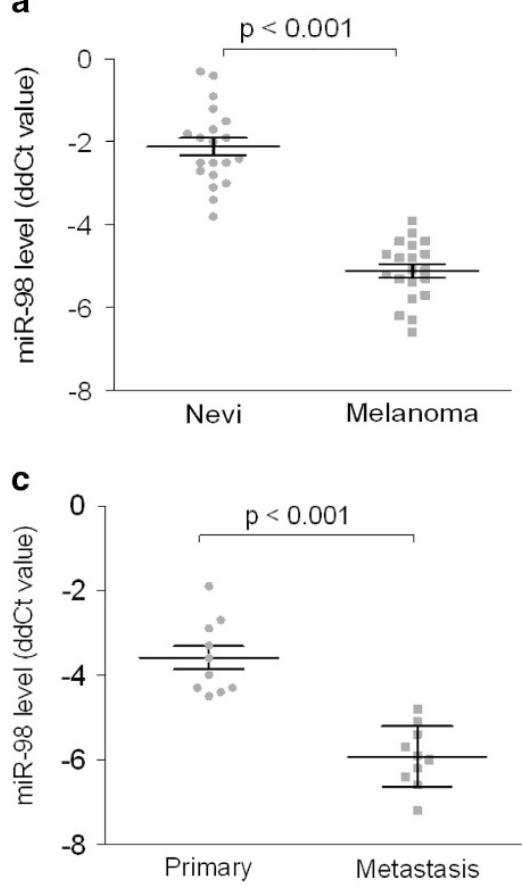

b

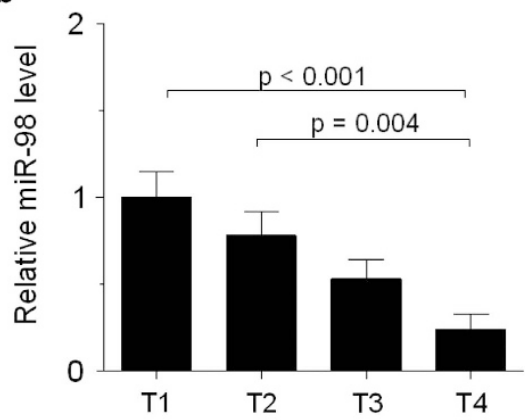

d

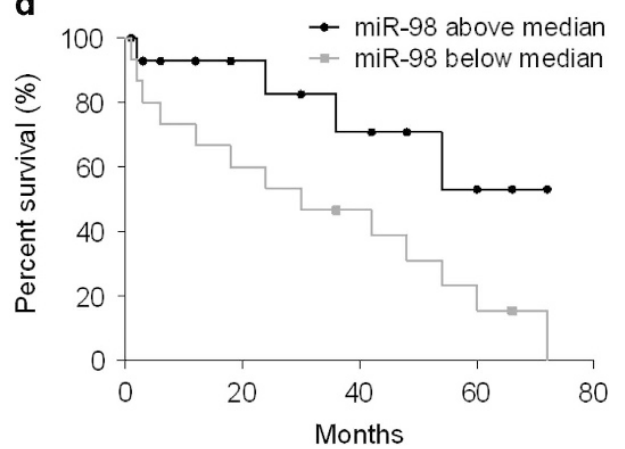

Figure 1 Reduced miR-98 expression marks the progression from primary to metastatic tumors and is associated with lower overall survival in melanoma patients. (a-c) MiR-98 levels were determined using quantitative reverse transcription-polymerase chain reaction (QRT-PCR). Data are presented as mean and s.d. (a) $N=24$ in each group; (b) $n=13,11,9$ and 15 in the T1, T2, T3 and T4 groups, respectively; (c) $n=15$ in each group; and (d) Kaplan-Meier survival curve, the dots in the figure indicate the censored cases. The log-rank test indicates that patients with miR-98 levels above the median had significantly higher survival rates than those with miR-98 levels below the median, $P=0.027$. 
two-sample $t$-tests. Comparisons between three or more groups were undertaken with analysis of variance with Fisher's least significant difference post hoc comparison. The survival rates were analyzed using Kaplan-Meier estimates and compared using the logrank test. Data were analyzed with SPSS 15.0 (SPSS, Chicago, IL, USA). All statistical analyses were two-sided, and $P$-values $<0.05$ were considered statistically significant.
RESULTS

Reduced miR-98 expression marks the progression from primary to metastatic tumors and is associated with lower overall survival in melanoma patients

Analysis of miR-98 levels in nevi and melanoma tissues revealed that it was significantly reduced in melanoma tissues $(P<0.001$, Figure 1a). As shown in Figure 1b, miR-98 levels a

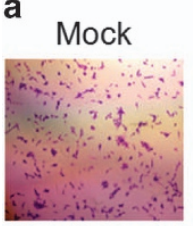

miRNA-NC
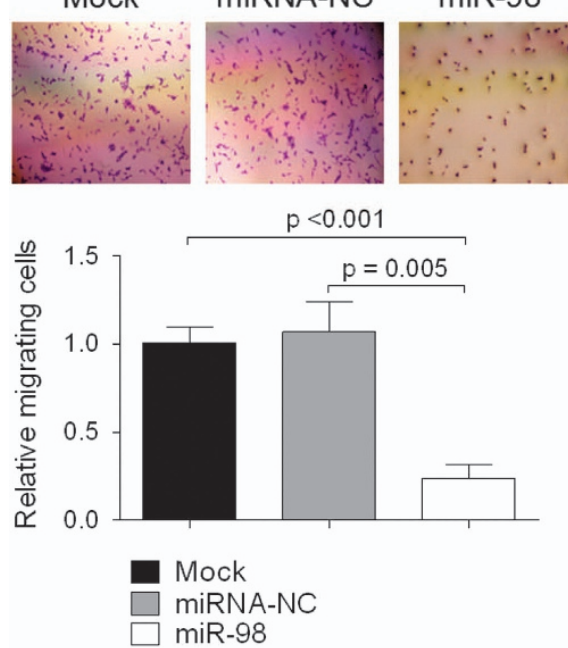

b
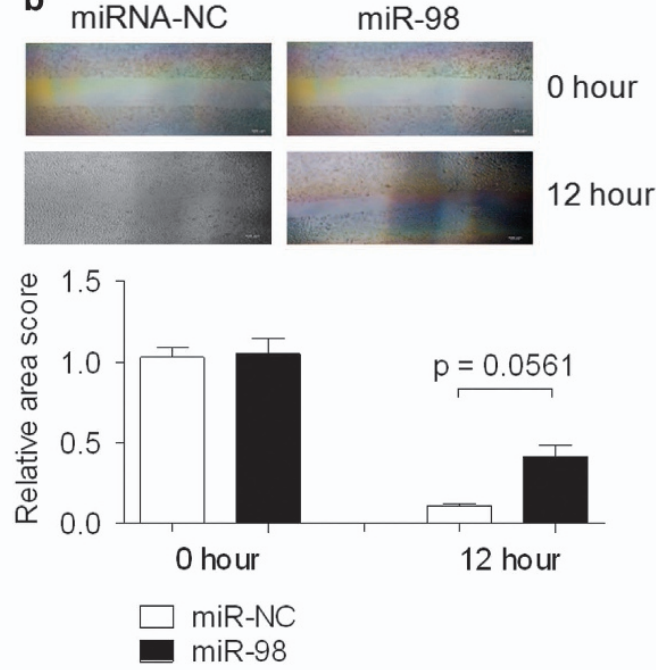

c
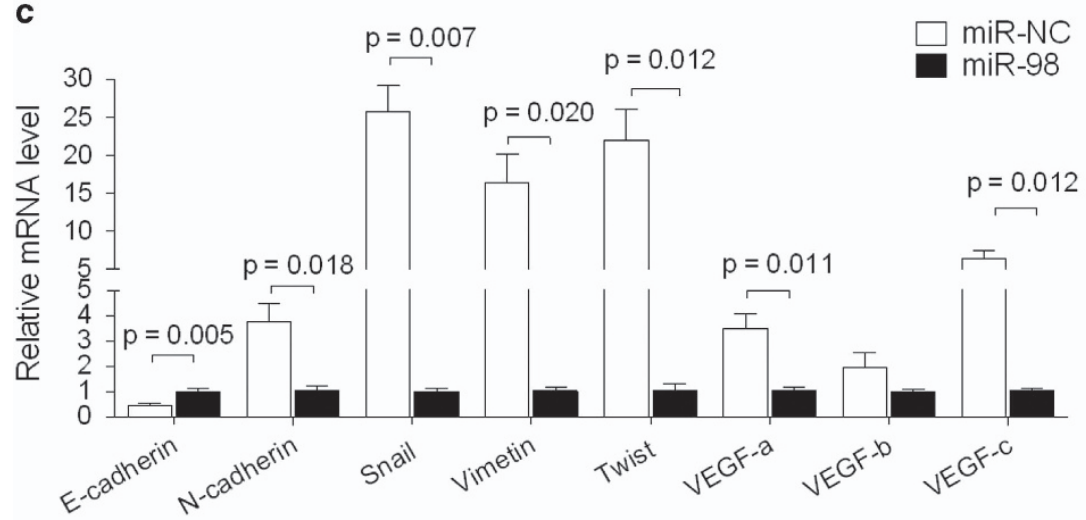

d
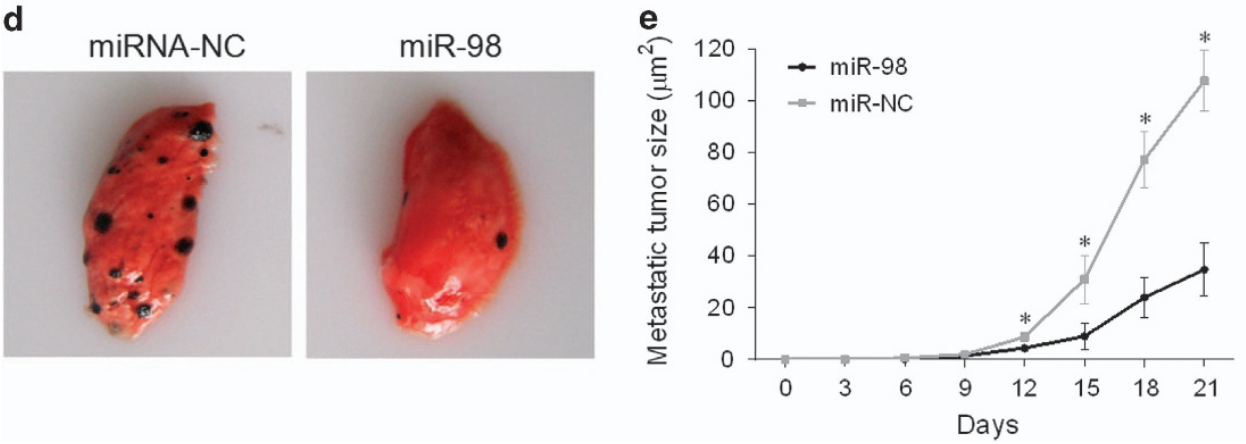

Figure 2 MiR-98 suppresses melanoma metastasis in vitro and in vivo. After B16-F1 cells were transfected with microRNA (miRNA)negative control (NC) or miR-98 for $36 \mathrm{~h}$, (a and b) cell migration and (c) expression of genes associated with cell migration were assessed. Data are presented as mean \pm s.d., $n=3$ in each group. (d) Representative gross image of lung metastases at day 21. (e) Metastatic tumor size of both miRNA negative control (miRNA-NC) and miR-98 groups over time. *A statistically significant difference between the miR-98 and miR-NC groups, $P \leqslant 0.011$ 
decreased with increased $\mathrm{T}$ stage; its expression in $\mathrm{T} 4$ tissues was significantly lower than that observed in $\mathrm{T} 1$ and $\mathrm{T} 2$ tissues $(P<0.001$ and $P=0.004$, respectively). Moreover, compared with primary tumors, miR-98 levels were significantly lower in metastatic tumors $(P<0.001$, Figure 1c). In addition, patients with miR-98 levels above the median had a significantly higher survival rate than those with miR-98 levels below the median $(P=0.027$, Figure 1d).

MiR-98 suppresses melanoma metastasis in vitro and in vivo The effects of miR-98 overexpression in B16-F1 cells were assessed. As shown in Figure 2a, significantly fewer migrating cells were observed in the miR-98 group compared with the mock $(P<0.001)$ and miRNA-NC $(P=0.005)$ groups. In addition, an increased area score was observed in the miR-98 group compared with the miR-NC group at $12 \mathrm{~h}(P=0.0561$, Figure $2 \mathrm{~b})$. Subsequent analysis of migration-associated genes in B16-F1 cells revealed a significant reduction in the mRNA levels of $\mathrm{N}$-cadherin $(P=0.018)$, snail $(P=0.007)$, vimentin $(P=0.02)$, twist $(P=0.012)$, VEGF-a $(P=0.011)$ and VEGF-c $(P=0.012)$ in the miR-98 group. In contrast, E-cadherin levels were significantly increased in the miR-98 group $(P=0.005$, Figure 2c).

In an in vivo model, the metastatic tumor size was increased over time in both the miR-98 and miR-NC groups (Figure $2 \mathrm{~d}$ ). However, the tumor size was significantly decreased by day 12 in the miR-98 group compared with the miR-NC group
$(P=0.009)$. This trend continued through the end of the study on day $21(P<0.001$, Figure $2 \mathrm{e})$.

\section{Identification of IL-6 as a direct target gene of miR-98}

To study the molecular mechanism by which miR-98 inhibits metastasis, we applied TargetScan prediction software (http:// www.targetscan.org/) and found an miR-98 binding site at $312-318$ in the $3^{\prime}$-UTR region of the $I L-6$ gene, which is conserved among mice, rats, humans and other mammals (Figure 3a). To verify whether $I L-6$ is an miR-98 target gene, we overexpressed miR-98 in B16-F1 cells and analyzed IL-6 reporter activity. The relative $3^{\prime}$-UTR luciferase activity was significantly decreased with miR-98 overexpression compared with miRNA-NC $(P<0.001$, Figure 3b). Moreover, luciferase activity was significantly increased when the cells were treated with anti-miR-98 compared with anti-miRNA-NC-treated cells (Figure 3b). The reporter activity of the mutant $3^{\prime}-\mathrm{UTR}$ was not altered with miR-98 or anti-miR-98 (Figure 3c).Western blot analysis also confirmed that miR-98 can inhibit IL-6 protein expression and that anti-miR-98 had relieved the effect of miR-98 on IL-6 (Figure 3d). In addition, secreted IL-6 was significantly decreased in the miR-98 group compared with the miRNA-NC group $(P<0.001)$; it was significantly increased in the anti-miR-98 group compared with the anti-miRNA-NC group $(P=0.001$, Figure $3 \mathrm{e})$. MiR-98 inhibits the protein expression and secretion of IL- 6 by binding its $3^{\prime}$-UTR.

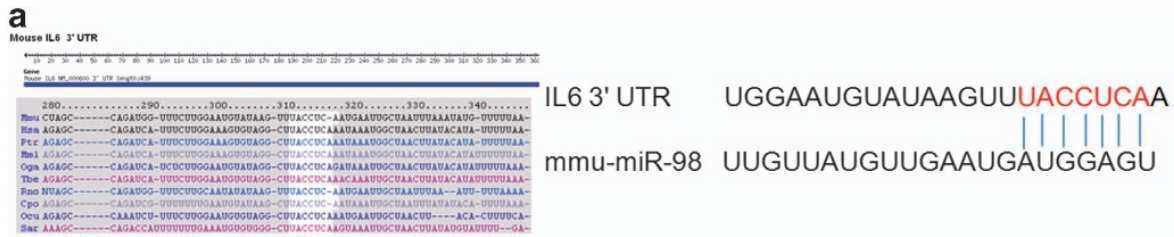

b

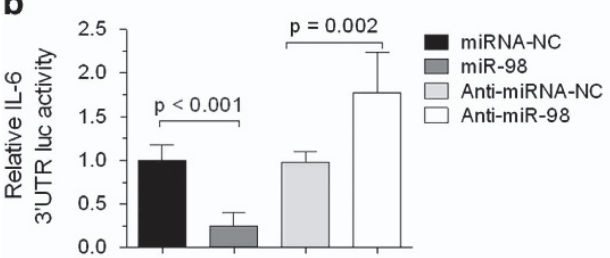

d

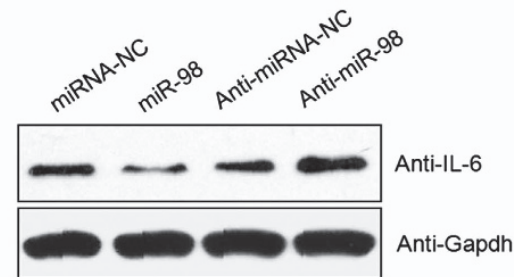

C

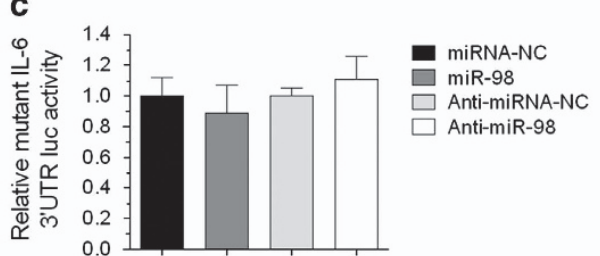

e

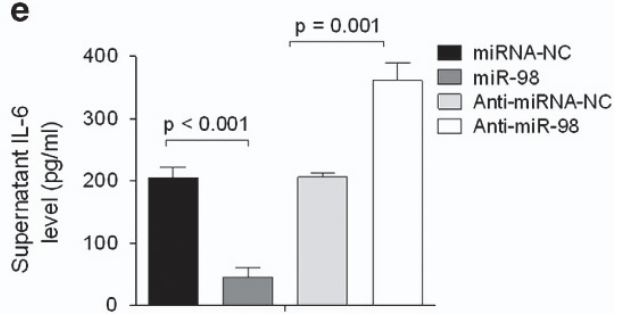

Figure 3 Identification of interleukin-6 (IL-6) as direct target gene of miR-98. (a) Putative miR-98 binding site within the 3'-untranslated regions ( $3^{\prime}$-UTR) of the IL-6 gene. ( $\mathbf{b}$ and $\mathbf{c}$ ) Relative luciferase activity of the (b) wild-type and (c) mutant IL-6 reporter after transfection of B16-F1 cells with miRNA-negative control (miRNA-NC), miR-98, anti-miRNA-NC and anti-miR-98. Data are presented as mean \pm s.d. ( $n=6$ in each group). (d) Western blot analysis of IL-6 protein levels after the indicated treatment. Glyceraldehyde 3-phosphate dehydrogenase (GAPDH) served as the loading control. (e) Secreted IL- 6 levels were determined by enzyme-linked immunosorbent assay (ELISA) after the indicated treatment. Data are presented as mean \pm s.d. ( $n=3$ in each group). 
IL-6 promotes melanoma metastasis in vitro and in vivo The protein levels of IL-6 were analyzed in a tissue array comprised of normal, melanoma and metastatic tissues by immunohistochemistry staining (Figure 4a). Analysis of the staining revealed that IL-6 expression was much higher in the metastatic samples compared with the control samples $(P=0.006$, Figure $4 \mathrm{~b})$.

The effects of IL-6 on B16-F1 cell migration were next evaluated in vitro and in vivo. As shown in Figure 4c, B16-F1 cell migration increased after treatment with IL-6. In addition, the metastatic tumor size was significantly greater after treatment with IL-6 at days $18(P=0.001)$ and $21(P=0.02$, Figures $4 \mathrm{~d}$ and e). IL-6 treatment also led to significantly increased mRNA levels of $\mathrm{N}$-cadherin $(P=0.014)$, snail $(P=0.045)$, vimentin $(P=0.022)$, twist $(P=0.034)$, VEGF-a $(P=0.027)$ and VEGF-c $(P=0.024)$, and decreased E-cadherin levels $(P=0.003$, Figure $4 \mathrm{f})$.

\section{MiR-98 is negatively regulated by IL-6 signaling through $\operatorname{lin} 28 B$}

We next determined the miR-98 levels after the cells were treated with IL-6. As shown in Figure 5a, treatment with IL-6 led to decreased miR-98 levels in a dose-dependent manner. Alternatively, significantly increased expression of miR-98 was observed over time after treatment with an IL-6-neutralizing antibody (Figure 5f). Increased miR-98 expression coincided with decreased secreted IL-6 protein levels (Figure 5g). Figure $6 \mathrm{~b}$ shows that the effects of IL- 6 on miR-98 levels were inhibited with siRNA-Stat3, but not with siRNA-NC. As shown in Figure $5 c$, the effects of IL- 6 were also blocked by the NF- $\kappa B$ a

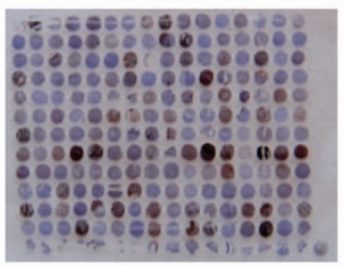

C

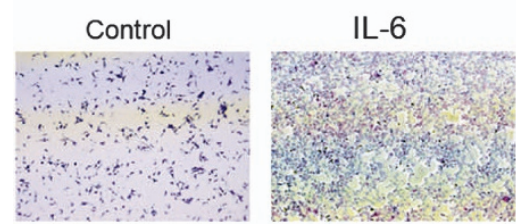

e

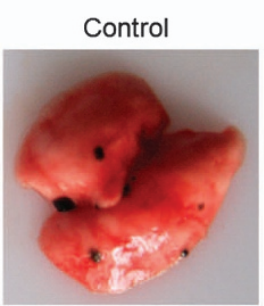

b
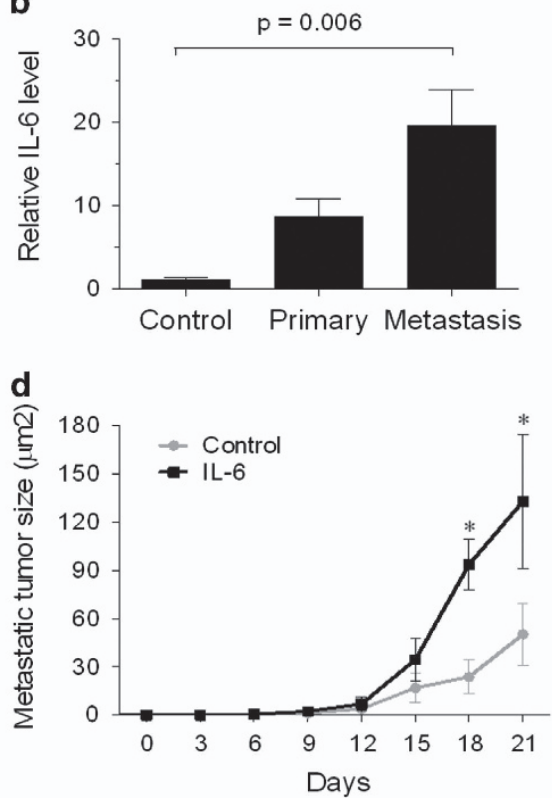

f

Figure 4 Interleukin-6 (IL-6) promotes melanoma metastasis in vitro and in vivo. (a) Representative immunohistochemical analysis of a tissue array for IL-6 expression. (b) Relative IL-6 protein expression in control, primary and metastatic tissues as determined by immunohistochemistry. Data are presented as mean \pm s.d. ( $n=8,15$ and 15 in control, primary and metastasis groups). (c) Respective image of B16-F1 migration after IL-6 treatment. (d) Metastatic tumor size of both the control and IL-6 groups over time. *A significant difference between the control and IL-6 groups ( $P=0.001$ and $P=0.020$ on days 18 and 21 , respectively). Data are presented as mean \pm s.d. ( $n=4$ in each group). (e) Representative gross image of lung metastases at day 21 . (f) Analysis of migration-associated gene expression after treatment with IL-6. *A significant difference between the control and IL- 6 groups, $P \leqslant 0.045$. 

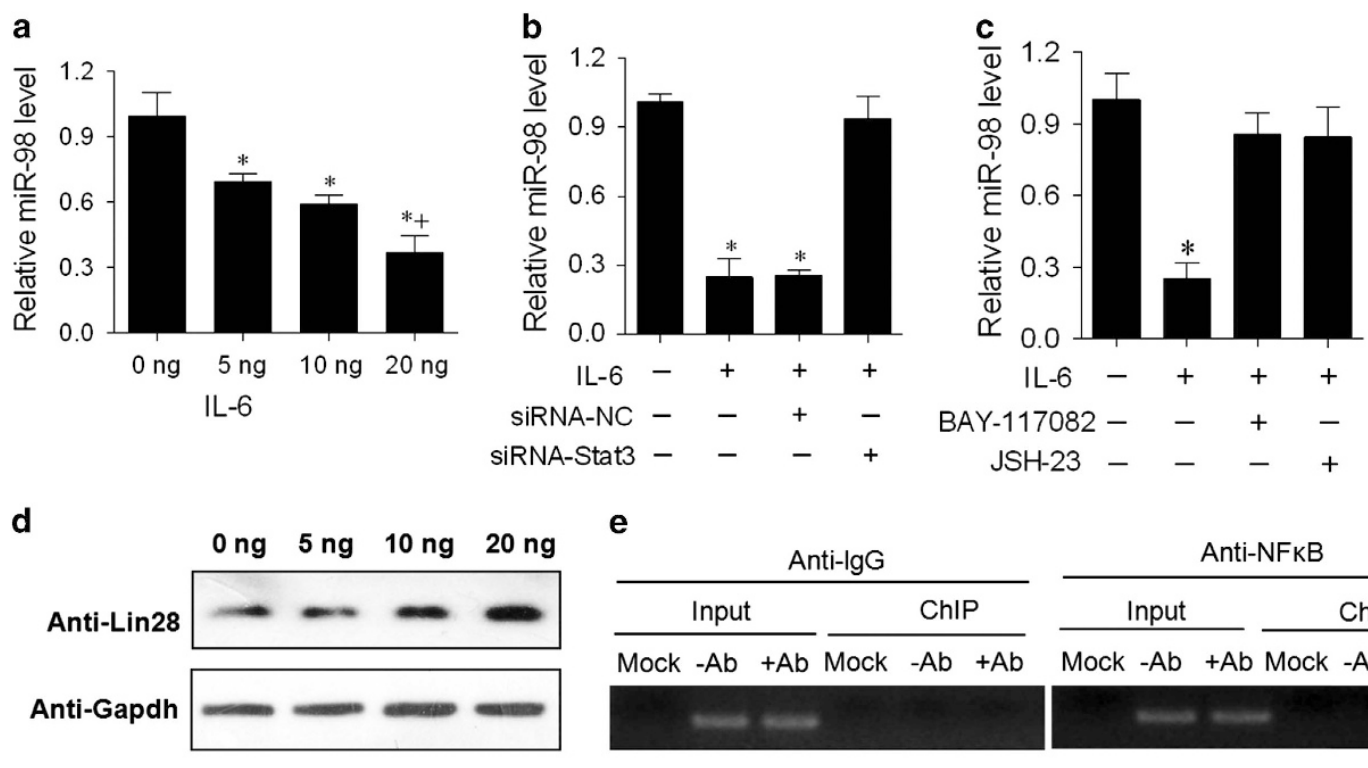

e
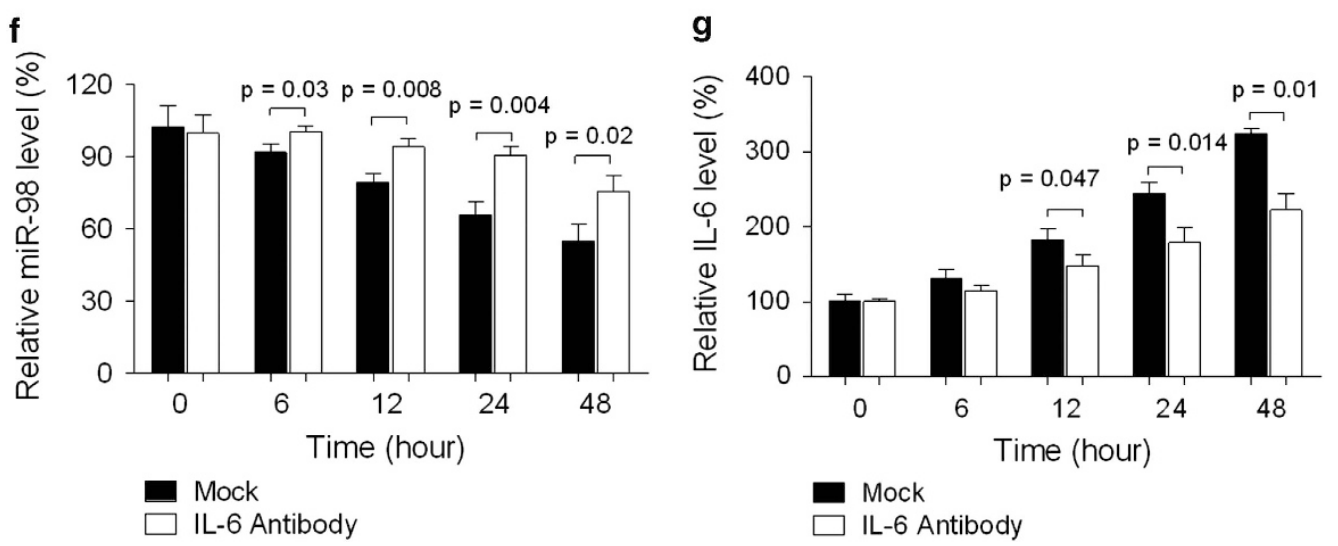

Figure 5 MiR-98 is negatively regulated by interleukin-6 (IL-6) signaling through lin28B. (a) Quantitative reverse transcription-polymerase chain reaction (RT-PCR) analysis of miR-98 levels in B16 cell after treatment with IL-6. (b) Quantitative RT-PCR analysis of miR-98 levels in signal transducer and activator of transcription 3 (Stat3) small interfering RNA (siRNA)-transfected B16 cells after treatment with IL-6. (c) Quantitative RT-PCR analysis of miR-98 levels in B16 cells after treatment with IL-6 and the nuclear factor-kB (NF-kB) inhibitors, BAY-117082 or JSH-23. (d) Western blot analysis of lin28B protein level after treatment with IL-6. (e) Chromatin immunoprecipitation analysis of lin28B promoter binding by NF-KB. (f) Relative miR-98 level in B16 cells after treatment with IL- 6 antibodies. (g) Relative secreted IL-6 level in B16 cell medium after treatment with IL- 6 antibodies. Data are presented as mean \pm s.d. ( $n=3$ for each group).

inhibitors, BAY-117082 and JSH-23. In addition, IL-6 induced lin28B (a negative regulator of miR-98) expression (Figure 5d). Chromatin immunoprecipitation analysis of the lin $28 B$ promoter revealed its interaction with NF-אB (Figure 5e).

\section{In vivo validation of the relationship between miR-98 and}

\section{IL-6 production}

The effect of miR-98 on IL- 6 and the subsequent NF- $\mathrm{kB}$ pathway was further evaluated in vivo using the metastatic tumor model used in Figure 2. The therapeutic effect of miR-98 application was validated once again (Figures $6 a-c$ ). The size of metastatic tumors in the miR-NC group continuously increased from day 15 until the end of the study, whereas the tumor size was significantly smaller in those treated with miR-98 from days 20 to $35 \quad(P \leqslant 0.018$,
Figure 6a). Representative lungs from both groups are shown in Figure $6 \mathrm{~b}$. The survival rate for the miR-98 group was higher than that observed for the miR-NC group, although it did not reach statistical significance (Figures $6 \mathrm{c}$, $P=0.076$ ). A concomitant increase in serum IL-6 levels was observed in the miR-NC group; however, no such increase was observed in the miR-98 group (Figure 6d). Significant differences between the two groups were observed on days 25 , 30 or $35(P \leqslant 0.006)$.

Analysis of Stat 3 and p 65 phosphorylation as well as lin28B mRNA levels at the end of the study was also undertaken. As shown in Figure 6e, the phosphorylation of both Stat3 and p65 was reduced in the miR-98 group compared with the controls. In addition, the lin28B mRNA levels in the miR-98 group was also decreased compared with the miR-NC group $(P=0.003)$. 
a

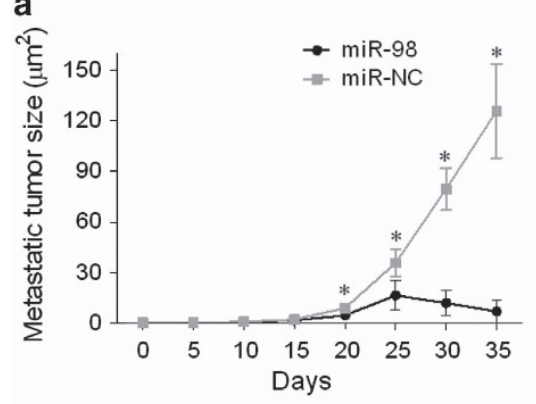

d

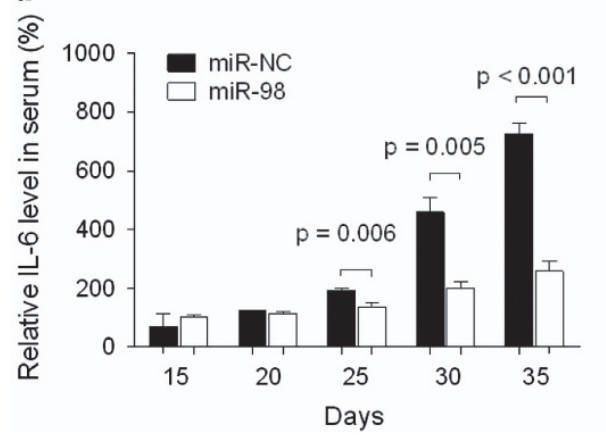

b

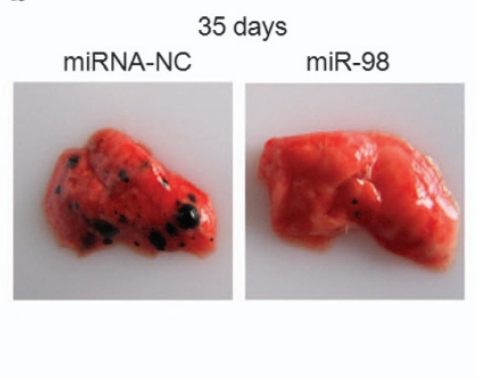

C

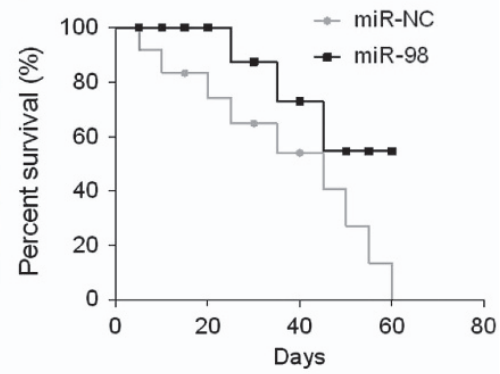

f

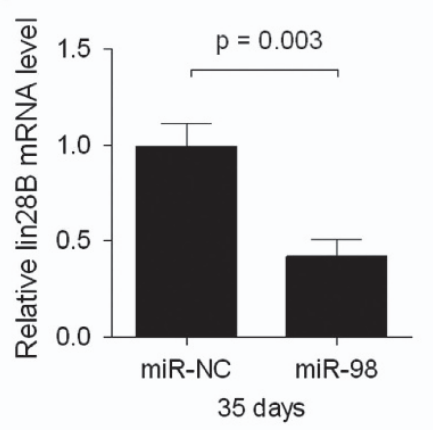

Figure 6 In vivo validation of the relationship between miR-98 and interleukin-6 (IL-6) production. (a) Metastatic tumor size of both the miRNA-negative control (miR-NC) and miR-98 groups over time. Data are presented as mean \pm s.d. ( $n=3$ in each group). ${ }^{*} \mathrm{~A}$ statistically significant difference between the miR-98 and miR-NC groups, $P \leqslant 0.018$. (b) Representative gross image of lung metastases at day 35 . (c) Kaplan-Meier survival curve, the dots in the figure indicate the censored cases. Log-rank tests indicate a significant difference between the groups, $P=0.076$. (d) Relative serum IL-6 levels after mice were treated with miRNA-NC or miR-98; $n=3$ for each group. (e) Western blot analysis of total Stat3 and p64 and phosphorylated Stat3 and p65 after 35 days treatment with miR-98 or miRNA-NC in tumor samples at day 35. (f) Relative lin28B mRNA levels in tumor samples at day 35 after mice were treated with miRNA-NC or miR-98. Data are presented as mean \pm s.d. ( $n=3$ in each group).

\section{DISCUSSION}

The prognosis of melanoma patients with regional or distant metastases remains poor ${ }^{5}$ due in part to limited prognostic markers and treatment options. Given the effects of miR-98 on cancer cell growth, survival and invasive potential, ${ }^{17,18}$ the present study sought to evaluate the levels of miR-98 in melanoma patients and its effects on migration and metastasis. In addition, the mechanism underlying the effects of miR-98 was explored. Downregulation of miR-98, a let-7 family member, was detected in human melanoma samples, and miR-98 inhibited melanoma metastasis in part through a novel miR-98-IL-6-negative feedback loop.

In the present study, reduced expression of miR-98 was observed with increasing melanoma stage. MiR-98 expression was downregulated in metastatic tumors compared with primary tumors. This is consistent with what has been observed in human lung adenocarcinoma cells. ${ }^{19}$ Moreover, altered miR-98 expression has been associated with the normal-tocancerous transition in endometrial cells. ${ }^{20}$ Further studies will determine the effects of silencing miR-98 in normal melanocytes to determine the effect of this change on cancer initiation.

Analysis of the effects of miR-98 on breast cancer cells revealed that it inhibited cell proliferation, survival, growth, invasion and angiogenesis. ${ }^{17}$ Reduced human esophageal squamous cell carcinoma migration and invasion was also observed with miR-98. ${ }^{21}$
These results are consistent with those observed in the present study where ectopic expression of miR-98 inhibited B16-F1 cell migration as well as in vivo metastasis and tumor growth. Similarly, we observed reduced expression of migration- and angiogenesis-associated gene expression with miR-98. However, the effects of miR-98 on angiogenesis were not directly assessed, but this will be the subject of further studies.

In the present study, IL-6, an immunomodulatory cytokine, was found to be a miR-98 target. In addition, it promoted tumor growth and invasion, which is consistent with previous studies. ${ }^{22-24}$ Increased serum levels of IL-6 were an independent prognostic biomarker of shorter overall survival in metastatic melanoma patients. ${ }^{25}$ Therefore, the reduced miR-98 levels in these patients will likely result in a concomitant increase in IL-6 levels and poorer prognosis. Further studies will evaluate the correlation between IL-6 and miR-98 levels in melanoma patients as well as the prognostic value of determining miR-98 levels in these patients. In addition, further tissue array analyses will be undertaken to determine the cell types that express IL- 6 in malignant melanoma (for example, tumor cells and/or surrounding immune cells).

In the present study, we found that IL- 6 also repressed miR-98 expression with a role for Stat ${ }^{26}$ and NF- $\mathrm{KB}^{27}$ signaling on IL-6 activity and expression, respectively. We analyzed 
miR-98 expression in the presence of Stat3 silencing as well as NF- $\kappa \mathrm{B}$ inhibitors.

The therapeutic potential of miR-98 was illustrated using an miR-98 mimic in the present study. Specifically, miR-98 mimics reduced tumor size as well as increased survival in a mouse model of metastatic melanoma, which is in agreement with that observed in prostate cancer cells. ${ }^{18}$ This is also consistent with studies that reported an increase in cisplatin sensitivity in resistant human lung adenocarcinoma cells. ${ }^{19,28}$ Further studies will evaluate the effects of miR-98 along with chemotherapies to determine if this miRNA can also increase melanoma sensitivity.

Although we show that IL-6 is a target of miR-98 and that miR-98 is negatively regulated by IL-6 via $\operatorname{lin} 28 \mathrm{~B}$, further studies will assess both miR-98 and IL-6 expression in patient tissues to assess their association. Furthermore, although this study focused on IL-6 signaling as a means by which miR-98 suppresses tumor metastasis and growth, the effects of miR-98 may also be mediated through additional mechanisms, which will be elucidated in further studies. Additional studies analyzing the therapeutic effects of miR-98 will be carried out using increased administration time and dosage. These studies will form the basis for further evaluation of the prognostic and therapeutic value of miR-98.

In conclusion, miR-98 is a suppressor of IL-6 signaling, forming a negative feedback loop to suppresses melanoma metastasis.

\section{CONFLICT OF INTEREST}

The authors declare no conflict of interest.

\section{ACKNOWLEDGEMENTS}

We thank the academic staff, An Hong and Zhi Shi, from the Jinan University for providing critical comments and technical support. We thank Guang Zhao for constructive suggestions and careful revisions to this manuscript. This work was supported by National Natural Science Foundation Projects (31000386 and 81370971), Guangdong Natural Science Funds for Distinguished Young Scholar (S2013050013880) and The Medical Science Youth Training Projects of PLA (13QNP077).

1 Tucker MA. Melanoma epidemiology. Hematol Oncol Clin N Am 2009; 23: 383-395.

2 Linos E, Swetter SM, Cockburn MG, Colditz GA, Clarke CA. Increasing burden of melanoma in the United States. J Invest Dermatol 2009; 129: 1666-1674.

3 Baade P, Coory M. Trends in melanoma mortality in Australia: 1950-2002 and their implications for melanoma control. Aust NZ J Public Health 2005; 29: 383-386.

4 Coory M, Baade P, Aitken J, Smithers M, McLeod GR, Ring I. Trends for in situ and invasive melanoma in Queensland, Australia, 1982-2002. Cancer Causes Control 2006; 17: 21-27.

5 Jemal A, Siegel R, Ward E, Hao Y, Xu J, Murray T et al. Cancer statistics, 2008. CA Cancer J Clin 2008; 58: 71-96.

6 Mueller DW, Bosserhoff AK. Role of miRNAs in the progression of malignant melanoma. Br J Cancer 2009; 101: 551-556.

7 Howell PM, Li X, Riker AI, Xi Y. MicroRNA in melanoma. Ochsner J 2010; 10: 83-92.

8 Lu J, Getz G, Miska EA, Alvarez-Saavedra E, Lamb J, Peck D et al. MicroRNA expression profiles classify human cancers. Nature 2005; 435 . 834-838.
9 Zhang L, Huang J, Yang N, Greshock J, Megraw MS, Giannakakis A et al. MicroRNAs exhibit high frequency genomic alterations in human cancer. Proc Natl Acad Sci USA 2006; 103: 9136-9141.

10 Mueller DW, Rehli M, Bosserhoff AK. MiRNA expression profiling in melanocytes and melanoma cell lines reveals miRNAs associated with formation and progression of malignant melanoma. J Invest Dermatol 2009; 129: 1740-1751.

11 Bemis LT, Chen R, Amato CM, Classen EH, Robinson SE, Coffey DG et al. MicroRNA-137 targets microphthalmia-associated transcription factor in melanoma cell lines. Cancer Res 2008; 68: 1362-1368.

12 Segura MF, Hanniford D, Menendez S, Reavie L, Zou X, Alvarez-Diaz S et al. Aberrant miR-182 expression promotes melanoma metastasis by repressing FOXO3 and microphthalmia-associated transcription factor. Proc Natl Acad Sci USA 2009; 106: 1814-1819.

13 Bar-Eli M. Searching for the 'melano-miRs': miR-214 drives melanoma metastasis. EMBO J 2011; 30: 1880-1881.

14 Schultz J, Lorenz P, Gross G, Ibrahim S, Kunz M. MicroRNA let-7b targets important cell cycle molecules in malignant melanoma cells and interferes with anchorage-independent growth. Cell Res 2008; 18: 549-557.

15 Fu TY, Chang CC, Lin CT, Lai CH, Peng SY, Ko YJ et al. Let-7b-mediated suppression of basigin expression and metastasis in mouse melanoma cells. Exp Cell Res 2011; 317: 445-451.

16 Schuchter LM. Review of the 2001 AJCC staging system for cutaneous malignant melanoma. Curr Oncol Rep 2001; 3: 332-337.

17 Siragam V, Rutnam ZJ, Yang W, Fang L, Luo L, Yang X et al. MicroRNA miR-98 inhibits tumor angiogenesis and invasion by targeting activin receptor-like kinase-4 and matrix metalloproteinase-11. Oncotarget 2012; 3: $1370-1385$.

18 Ting HJ, Messing J, Yasmin-Karim S, Lee YF. Identification of microRNA-98 as a therapeutic target inhibiting prostate cancer growth and a biomarker induced by vitamin D. J Biol Chem 2013; 288: 1-9.

19 Xiang Q, Tang H, Yu J, Yin J, Yang X, Lei X. MicroRNA-98 sensitizes cisplatin-resistant human lung adenocarcinoma cells by up-regulation of HMGA2. Pharmazie 2013; 68: 274-281.

20 Panda H, Chuang TD, Luo X, Chegini N. Endometrial miR-181a and miR-98 expression is altered during transition from normal into cancerous state and target PGR, PGRMC1, CYP19A1, DDX3X, and TIMP3. J Clin Endocrinol Metab 2012; 97: E1316-E1326.

21 Huang SD, Yuan Y, Zhuang CW, Li BL, Gong DJ, Wang SG et al. MicroRNA-98 and microRNA-214 post-transcriptionally regulate enhancer of zeste homolog 2 and inhibit migration and invasion in human esophageal squamous cell carcinoma. Mol Cancer 2012; 11: 51.

22 Hoejberg L, Bastholt L, Schmidt H. Interleukin-6 and melanoma. Melanoma Res 2012; 22: 327-333.

23 Naugler WE, Karin M. The wolf in sheep's clothing: the role of interleukin-6 in immunity, inflammation and cancer. Trends Mol Med 2008; 14: 109-119.

24 Jostock T, Müllberg J, Ozbek S, Atreya R, Blinn G, Voltz N et al. Soluble gp130 is the natural inhibitor of soluble interleukin-6 receptor transsignaling responses. Eur J Biochem 2001; 268: 160-167.

25 Hoejberg L, Bastholt L, Johansen JS, Christensen IJ, Gehl J, Schmidt H. Serum interleukin- 6 as a prognostic biomarker in patients with metastatic melanoma. Melanoma Res 2012; 22: 287-293.

26 Terai M, Eto M, Young GD, Berd D, Mastrangelo MJ, Tamura Y et al. Interleukin 6 mediates production of interleukin 10 in metastatic melanoma. Cancer Immunol Immunother 2012; 61: 145-155.

27 Karst AM, Gao K, Nelson CC, Li G. Nuclear factor kappa B subunit p50 promotes melanoma angiogeensis by upregulating interleukin 6 expression. Int J Cancer 2009; 124: 494-507.

28 Zhang S, Zhang C, Li Y, Wang P, Yue Z, Xie S. miR-98 regulates cisplatininduced A549 cell death by inhibiting TP53 pathway. Biomed Pharmacother 2011 ; 65 : 436-442.

This work is licensed under a Creative Commons Attribution-NonCommercial-ShareAlike 3.0 Unported License. The images or other third party material in this article are included in the article's Creative Commons license, unless indicated otherwise in the credit line; if the material is not included under the Creative Commons license, users will need to obtain permission from the license holder to reproduce the material. To view a copy of this license, visit http:// creativecommons.org/licenses/by-nc-sa/3.0/ 